Research Article

\title{
Fractal Analysis of Fragmentation Distribution of Rockbursts Induced by Low-Frequency Seismic Disturbances
}

\author{
Lihua Hu $\mathbb{D}^{1,2}$ Zhenghu Zhang $\mathbb{D}^{1},{ }^{1}$ Xin Liang, ${ }^{1}$ and Chunan Tang ${ }^{1}$ \\ ${ }^{1}$ Sate Key Laboratory of Coastal and Offshore Engineering, Dalian University of Technology, Dalian, Liaoning 116024, China \\ ${ }^{2}$ Key Laboratory of Disaster Prevention and Structural Safety of Ministry of Education, \\ School of Civil and Architecture Engineering, Guangxi University, Nanning, Guangxi 530004, China \\ Correspondence should be addressed to Zhenghu Zhang; zhenghuzhang@dlut.edu.cn
}

Received 12 December 2020; Revised 23 January 2021; Accepted 31 January 2021; Published 13 February 2021

Academic Editor: Pingye Guo

Copyright (C) 2021 Lihua Hu et al. This is an open access article distributed under the Creative Commons Attribution License, which permits unrestricted use, distribution, and reproduction in any medium, provided the original work is properly cited.

Low-frequency seismic disturbances frequently induce violent rockburst hazards, seriously threatening the safety of deep excavation and mining engineering. To investigate the characteristics and mechanisms of rockbursts induced by seismic disturbances, in this study a series of true triaxial experiments, including the moderate seismically induced, the weak seismically induced, and the self-initiated rockburst experiments under different conditions were conducted. The fractal geometry theory was applied to study rockbursts and the fractal dimensions of fragmentation distribution of different types of rockbursts were calculated. The results show that the fragmentation distributions of both the seismically induced and self-initiated rockbursts exhibit fractal behaviors. For the moderate seismically induced rockbursts, as the static stresses (i.e., the maximum and minimum static stresses) and disturbance amplitude increase, the fractal dimension increases, whereas, as the disturbance frequency increases, the fractal dimension decreases first and then increases. Under similar static loading conditions, the moderate seismically induced rockbursts have the largest fractal dimension, followed by the self-initiated rockbursts, and the weak seismically induced rockbursts have the smallest fractal dimension. There is a linear relationship between the average fractal dimension and kinetic energy of these rockbursts, implying that the fractal dimension can serve as an indicator for estimating rockburst intensity. Furthermore, from a fractal point of view, the energy input, dissipation, and release of these rockbursts are all linear processes.

\section{Introduction}

In recent decades, with the worldwide expansion of mining and civil engineering to the deep ground, many large deep-buried tunnels/openings have been built [1]. Excavation of deep hard rocks causes the near-boundary rock mass to be in a high-stress state. Meanwhile, there are many dynamic disturbances during the excavation. Under the combined effects of high static stress and dynamic disturbances, violent rockburst disasters are frequently encountered [2-11]. The rockbursts caused by dynamic disturbances often bring about equipment damage, casualties, and significant economic losses.

Due to the high incidence and dangerousness, the rockburst hazards caused by dynamic disturbances have aroused widespread concern of researchers. There are many related terms for rockbursts caused by dynamic disturbances, for example, "remotely triggered rockbursts $[6,8]$," "dynamically induced/triggered rockbursts $[2,4,5,9,10]$," "impact rockbursts [3]," and "seismically induced/triggered rockbursts [12]." The common feature of these rockbursts is that the rock mass is subjected to coupled static and dynamic loading [7]. The dynamic disturbances causing rockbursts can be high strain rate shock waves from blasting [7] or lowfrequency seismic waves/disturbances (or analogous stress waves $[2-6,8,9])$. There are many sources of the seismic disturbances in deep engineering, such as natural seismic events, stress waves attenuated from remote blasting, seismic events caused by fault-slip, rockbursts in nearby excavation, and ground vibration caused by huge vehicles [6, 8-10]. The low-frequency seismic disturbances have a long propagation 
distance and they carry a large amount of energy. Therefore, rockbursts caused by low-frequency seismic disturbances are the most common. In fact, a rockburst is defined by [12] as "damage to an excavation that occurs in a sudden and violent manner and is associated with a seismic event." In this study, we focus on the seismically induced rockbursts. Various methods, such as analytical $[3,4,7]$, experimental [2-6, 8-10], and numerical methods [4, 5] have been used to investigate the seismically induced rockbursts. However, due to the high complexity of the seismically induced rockbursts, the driving mechanisms are still not clear and effective control of them is a challenge.

A seismically induced rockburst is a complex dynamic instability phenomenon, which involves the processes of cracking, damage accumulation, and energy dissipation and release of rocks. Because rocks are geomaterials with good self-similar characteristics, their fracturing and failure processes exhibit statistic self-similar fractal behavior. The fractal theory is widely applied to study rock failures $[13,14]$ and rockbursts [15-26]. For example, fractal analyses on characteristics of rockburst cracks $[16,20]$, acoustic emissions (AE) [21, 24-26], and sounds [22] have been performed by researchers. Since the fragments generated from rockbursts contain a variety of information about the rockburst process and can reflect the mechanism of rockbursts, fractal studies have also been conducted on the fragment geometric characteristics and the fragmentation distribution of rockbursts $[15,17-19,23]$. In general, previous fractal studies focus on rockbursts without dynamic disturbances (i.e., the self-initiated rockbursts [12]). Fractal analyses on the fragmentation distribution of the seismically induced rockbursts have been rarely reported $[19,23]$. Moreover, previous studies do not link the fractal dimension and related physical quantities (e.g., the kinetic energy) of different types of rockbursts, which is urgent for the practical application of fractal theory in predicting rockbursts. Therefore, further study is needed to investigate the fractal characteristics of fragmentation distribution of the seismically induced rockbursts.

In this study, the fractal geometry theory was applied to investigate the characteristics and mechanisms of the seismically induced rockbursts. The experiments of seismically induced and self-initiated rockbursts were carried out by a novel servo control dynamic true triaxial rockburst testing system. The fragments generated in rockburst experiments were collected, sieved, and classified. The fractal dimensions of fragmentation distribution of different types of rockbursts were calculated according to the mass-equivalent size relationship. The effects of static and dynamic loading conditions on fractal dimensions of the seismically induced rockbursts were studied. By comparing the fractal dimensions, characteristics and mechanisms of different types of rockbursts were investigated. Moreover, the relationship between the fractal dimension and kinetic energy of different types of rockbursts was established.

\section{Materials and Methods}

2.1. Rock Sample Preparation. Rockbursts usually occur in deep hard brittle rocks. Granite and granodiorite are frequently encountered during the excavation of deep tunnels in China. Therefore, a typical hard brittle coarsegrained granodiorite is used for rockburst experiments. The size of rectangular prism rock samples is $100 \mathrm{~mm}(x-$ direction $) \times 100 \mathrm{~mm} \quad(y$-direction $) \times 200 \mathrm{~mm} \quad(z$-direction $)$. The integrity and homogeneity of the rock samples are relatively good. The tested basic physical and mechanical properties of the samples are as follows $[4,9]$ : the density is $2665 \mathrm{~kg} / \mathrm{m}^{3}$, the $P$ wave velocity is $5.2 \mathrm{~km} / \mathrm{s}$, Young's modulus is $30.55 \mathrm{GPa}$, Poisson's ratio is 0.26 , and the uniaxial compressive strength (UCS) is $180 \mathrm{MPa}$.

2.2. True Triaxial Experiments of the Seismically Induced Rockbursts. The true triaxial rockburst testing system developed at Guangxi University, China $[9,27,28]$, is used to conduct rockburst experiments (Figure 1). The rockburst testing system consists of a true triaxial testing machine, a high-speed camera system, an acoustic emission (AE) system, a control system, and a data acquisition system. The testing machine is an integral frame type press machine, which can independently load and unload in three mutually perpendicular directions, and conventional rock mechanics tests, such as uniaxial/triaxial compression, tensile, and shear tests can be performed with this testing machine. A specific function of keeping a rock sample in the state of "one face free and the other five faces loaded" is designed for the testing machine to simulate rockbursts $[9,27,28]$. A series of self-initiated rockburst experiments have been performed by the researchers [22, 27, 28].

In order to simulate the seismically induced rockbursts, the testing machine is also equipped with a dynamic disturbance system in three directions (Figure 1(b)). A variety of disturbance waveforms are available $[4,5,9]$. The in situ monitoring and previous studies $[5,8-10,29]$ show that the frequency of the seismic disturbances inducing rockbursts is low (e.g., several to tens of $\mathrm{Hz}$ ), and the induced dynamic stress increment (i.e., the amplitude) is in the range of 0-30 MPa. Therefore, cyclic loads with frequencies of $0.2-15 \mathrm{~Hz}$ and amplitudes of $2-40 \mathrm{MPa}$ are used in the experiments to simulate the seismic disturbances. Among them, the cyclic load with frequencies of $0.2-3 \mathrm{~Hz}$ and amplitudes of $10-40 \mathrm{MPa}$ is used to simulate a relatively intense seismic disturbance (termed as moderate seismic disturbance), and the cyclic load with a frequency of $15 \mathrm{~Hz}$ and amplitude of $2 \mathrm{MPa}$ is used to simulate a weak seismic disturbance.

The seismically induced rockbursts are failures of highly stressed rocks induced by seismic disturbances, that is, rock failures under coupled static and dynamic loading. The test processes of the seismically induced rockbursts are summarized as follows $[4,5,9]$ :

(1) Simulation of initial in situ stress state: Maintain one face of the rock sample free and apply a preset true triaxial stress state to the rock sample;

(2) Simulation of stress concentration after excavation: Maintain the lateral (the $x$ - and $y$-direction) stresses unchanged and gradually increase the vertical $(z$ - 


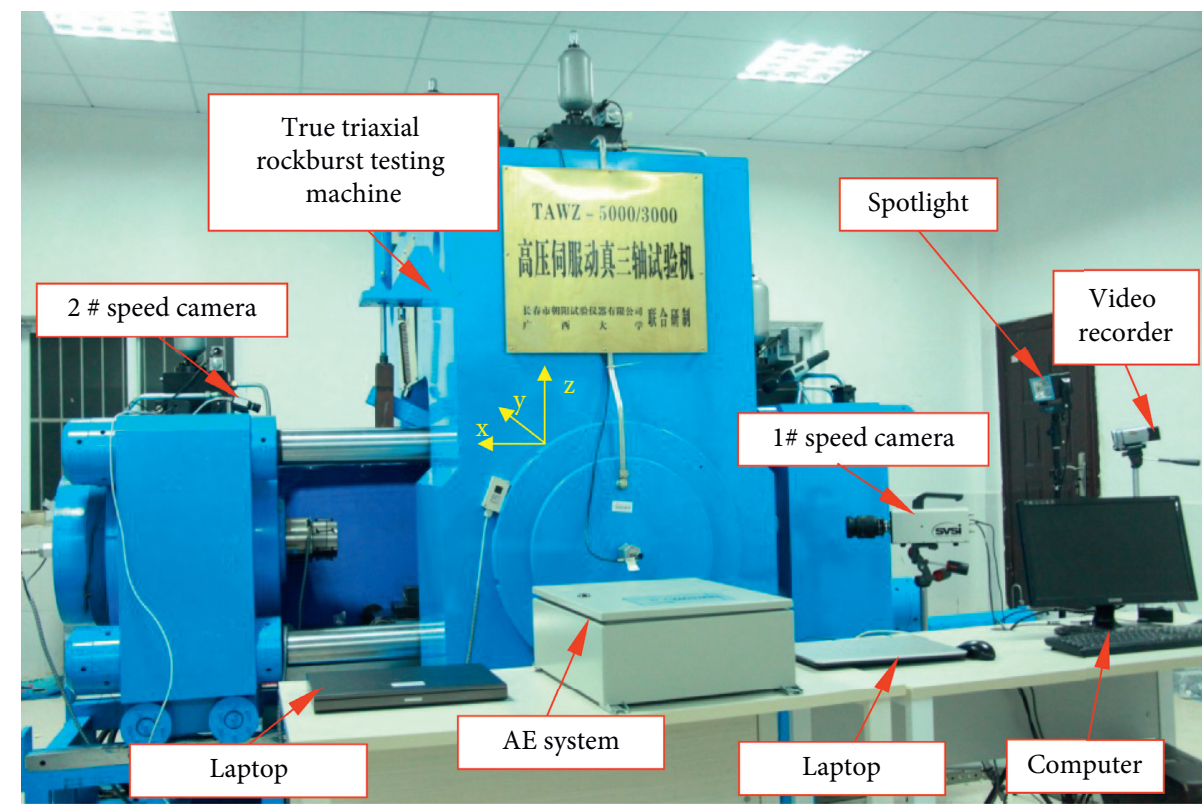

(a)
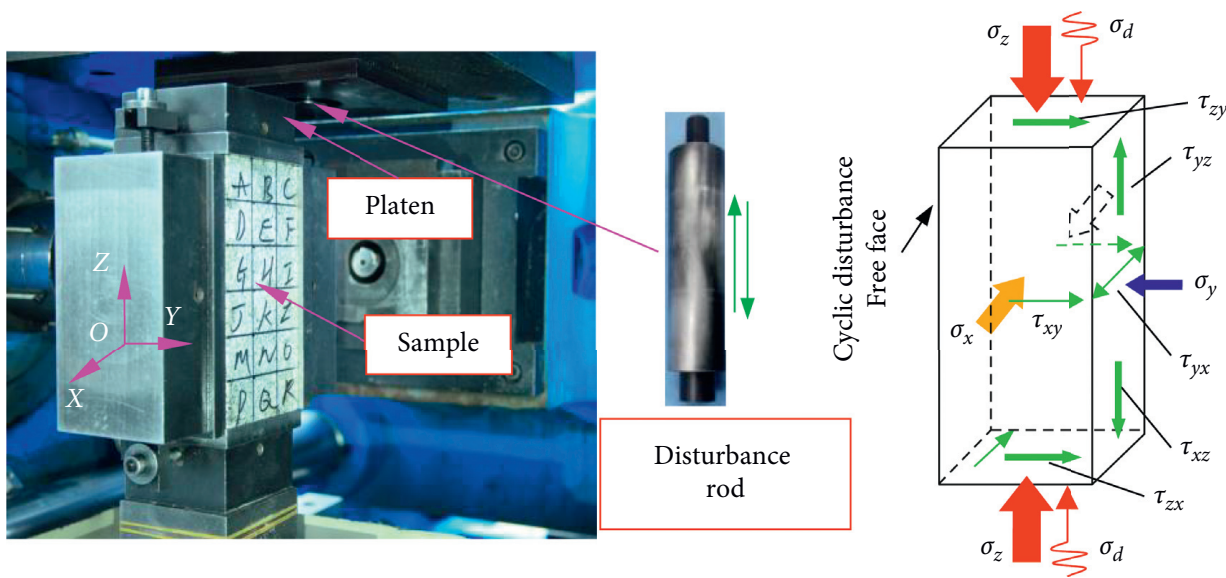

(b)

FIGURE 1: True triaxial experiments of the seismically induced rockbursts (modified after [5, 9]): (a) the true triaxial rockburst testing system, and (b) the loading configuration and the stress state of the rock sample, where $\sigma_{x}, \sigma_{y}$, and $\sigma_{z}$ are the intermediate, minimum, and maximum static stresses acting on the rock sample; $\tau_{x y}, \tau_{y z}$, and $\tau_{z x}$ are the shear stresses; and $\sigma_{d}$ is the seismic disturbance simulated by low-frequency cyclic disturbance.

direction) stress to a predetermined high stress at a rate of $0.5 \mathrm{MPa} / \mathrm{s}$;

(3) Simulation of seismic disturbance loading: A cyclic load is applied in the maximum static stress direction (z-direction) until a rockburst occurs or the disturbance duration reaches 15 minutes.

After rockburst experiments, the fragments generated are collected, classified, and measured, so as to study the characteristics of rockburst fragments.

2.3. Testing Design. The testing design is listed in Table 1. Different test groups are designed to study the effects of various static stresses and disturbance characteristics on rockbursts. For a weak seismically induced rockburst, it is essentially a dynamic failure of critically stressed rock triggered by a rather small disturbance [5]. The purpose of static loading is to reach a critical stress state of the rock. It is difficult to compare the critical stress states of rock under different static stress states. Therefore, only two groups of tests with different minimum static stresses are considered. The effect of disturbance on the weak seismically induced rockbursts is to activate and aggravate the rock damage, and the induced stress increment and the input energy to the rock by the disturbance are very small. Therefore, the frequency and amplitude of the weak seismic disturbance are set as constant values. Furthermore, self-initiated rockburst experiments under similar static conditions are conducted for contrast. 
TABLE 1: Testing design of true triaxial rockburst experiments.

\begin{tabular}{|c|c|c|c|c|c|c|c|}
\hline \multirow{2}{*}{ Test groups } & \multirow{2}{*}{ Specimen number } & \multicolumn{3}{|c|}{ Static stress } & \multicolumn{2}{|c|}{ Cyclic disturbance } & \multirow{2}{*}{ Rockburst } \\
\hline & & $\sigma_{x}(\mathrm{MPa})$ & $\sigma_{y}(\mathrm{MPa})$ & $\sigma_{z}(\mathrm{MPa})$ & $\Delta \sigma(\mathrm{MPa})$ & $f(\mathrm{~Hz})$ & \\
\hline Self-initiated rockbursts & SD-1 & 30 & 5 & 260 & - & - & Yes \\
\hline \multirow{16}{*}{ Moderate seismically induced rockbursts } & CD-Z-1 & 30 & 5 & 190 & 30 & 2.0 & No \\
\hline & $\mathrm{CD}-\mathrm{Z}-2$ & 30 & 5 & 210 & 30 & 2.0 & No \\
\hline & $\mathrm{CD}-\mathrm{Z}-3$ & 30 & 5 & 220 & 30 & 2.0 & Yes \\
\hline & CD-Z-4 & 30 & 5 & 240 & 30 & 2.0 & Yes \\
\hline & $\mathrm{CD}-\mathrm{Y}-1$ & 30 & 1 & 220 & 30 & 2.0 & Yes \\
\hline & CD-Y-2 & 30 & 2 & 220 & 30 & 2.0 & Yes \\
\hline & CD-Y-3 & 30 & 3 & 220 & 30 & 2.0 & No \\
\hline & CD-Y-4 & 30 & 5 & 220 & 30 & 20 & No \\
\hline & CD-A-1 & 30 & 5 & 220 & 10 & 2.0 & No \\
\hline & CD-A-2 & 30 & 5 & 220 & 20 & 2.0 & No \\
\hline & CD-A-3 & 30 & 5 & 220 & 30 & 2.0 & Yes \\
\hline & CD-A-4 & 30 & 5 & 220 & 40 & 2.0 & Yes \\
\hline & CD-F-1 & 30 & 5 & 220 & 30 & 0.2 & Yes \\
\hline & $\mathrm{CD}-\mathrm{F}-2$ & 30 & 5 & 220 & 30 & 1.0 & Yes \\
\hline & CD-F-3 & 30 & 5 & 220 & 30 & 2.0 & Yes \\
\hline & CD-F-4 & 30 & 5 & 220 & 30 & 3.0 & Yes \\
\hline \multirow[t]{2}{*}{ Weak seismically induced rockbursts } & WD-1 & 30 & 5 & 244 & 2 & 15.0 & Yes \\
\hline & WD-2 & 30 & 10 & 277 & 2 & 15.0 & Yes \\
\hline
\end{tabular}

\section{Application of Fractal Theory in Studying Rockbursts}

Rocks are geomaterials with micropores, cracks, and macroscale geological structures (e.g., joints, faults, and weak sides). Therefore, at different scales, the mechanical behaviors of rocks exhibit uncertainty and irregularity. The classical mathematical and mechanical models are powerless in dealing with the irregularity of rock behaviors. Fortunately, the development of the fractal geometry theory provides an available method for studying the complex mechanical behaviors of rock materials. Fractal geometry theory is proposed by Mandelbrot in the 1980s [30], which is different from the traditional Euclidean geometry system. The fractal geometry theory provides an effective quantitative description for complex, irregular, and seemingly disordered phenomena and systems that are widespread in natural and social activities. Irregular curves or shapes with self-similar and irregular figures with self-inverse characteristics are the main research objects of fractal geometry. Although the mechanical behaviors of rock materials are complex, there are still statistical self-similar characteristics. Therefore, fractal geometry is widely used in rock mechanics, such as in the study of rock fracturing, crack propagation, roughness of rock joints, fragmentation distribution, and rockbursts. In fact, a discipline of fractal rock mechanics has formed now [31].

Rockbursts are violent failure forms of hard brittle rocks, which exhibit fractal behaviors in many aspects (Figure 2), such as microfracturing (Figure 2(a)) [32], fragmentation distribution of rock samples in the laboratory (Figure 2(b)) [17], and fragmentation distribution on the project site (Figure 2(c)) [33]. The fracturing process of rockbursts can be illustrated using the fractal geometry of Sierpinski gasket (Figure 2(d)).
In previous studies, researchers have conducted fractal analyses on rockbursts from the perspectives of microfracturing and crack propagation $[16,18,20]$, fragments $[15,17-19,23]$, and AE $[21,22,24]$. Among these studies, since rockburst fragments are easy to collect and analyze, it is an effective way to study rockburst fractal characteristics from the aspect of fragments. The fractal characteristics of rockburst fragments include the fragment geometric features (e.g. the length-width and width-thickness ratios) and fragmentation distribution. Research results have shown that the fractal dimensions of geometric features of rockburst fragments calculated by different methods are very different and difficult to compare [17]. The fragmentation distribution contains a variety of important information about rockbursts, such as fracturing, damage accumulation, energy dissipation, and intensity (i.e., the kinetic energy), and the calculation methods of the fractal dimension of fragmentation distribution are unified $[5,15]$. It is an effective way to investigate the fractal characteristics of rockbursts from the perspective of fragmentation distribution.

The fractal dimension of fragmentation distribution of rockbursts is affected by the types of rockbursts (e.g., the seismically induced or the self-initiated rockbursts), and the mechanical conditions of rockbursts (e.g., the coupled static and disturbance conditions of the moderate seismically induced rockbursts). Furthermore, in order to make the fractal dimension have a physical meaning and reveal the rockburst mechanisms, it is necessary to establish the relationship between the calculated fractal dimension and important rockburst physical quantities (e.g., the kinetic energy).

In the following sections, we will calculate the fractal dimensions of fragmentation distribution of the self-initiated and the seismically induced rockbursts, study the 


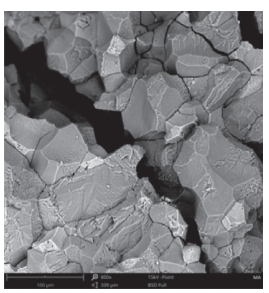

(a)

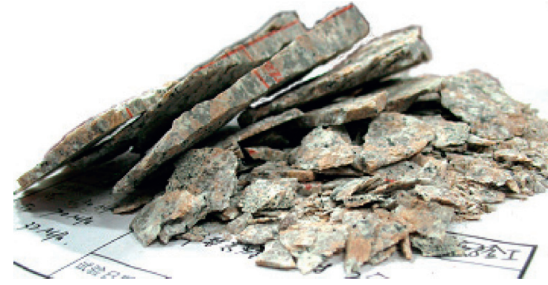

(b)

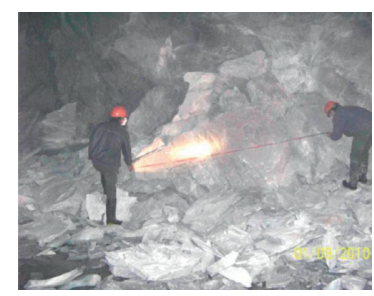

(c)

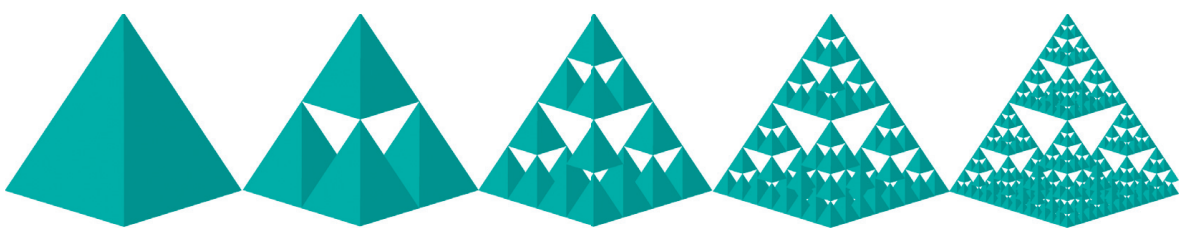

(d)

FIGURE 2: Fractal characteristics of rockbursts at different scales: (a) microcracking indicated by SEM image [32], (b) fragmentation distribution of rockburst sample fragments [17], (c) fragmentation distribution of the ejected fragments of an in situ rockburst [33], and (d) illustration of rockburst fracturing process with the Sierpinski gasket.

influences of different factors on fractal dimensions, and establish a quantitative relationship between the fractal dimension and rockburst kinetic energy.

\section{Fractal Analysis of Fragmentation Distribution of the Seismically Induced Rockbursts}

4.1. Equivalent Size of Rockburst Fragments. Figure 3 shows the fragmentation distribution of a typical seismically induced rockburst (CD-Z-3). It can be seen that the fragments are different in shape and size. In this study, we propose a new method for calculating the equivalent size to quantitatively describe the geometric and size characteristics of rockburst fragments. In general, for small fragments (e.g., maximum size less than $10 \mathrm{~mm}$ ), it is difficult to directly measure their sizes in three directions, and they can be sieved to estimate their equivalent sizes. For fragments larger than $10 \mathrm{~mm}$, it is easy to measure their sizes in three directions. Considering that the fragments are irregularly shaped, we define the largest dimensions of the fragments in three directions from large to small as length, width, and thickness.

In order to calculate the equivalent size, the fragments are sieved using sieves with diameters of $0.075,0.150,0.300$, $0.600,1.180,2.360,4.750$, and $9.500 \mathrm{~mm}$. Fragments with a maximum size less than $9.50 \mathrm{~mm}$ sequentially fall on each level of the sieve. For these fragments, the average size of diameters of the current and upper level sieves is taken as the equivalent size of fragments falling on a certain sieve.

For fragments with a maximum size larger than $9.50 \mathrm{~mm}$, in previous studies $[15,17,19,23]$, the equivalent sizes of these fragments are usually calculated according to the volume equivalent principle; that is, the side length of an equal volume cube is taken as the equivalent size. However, this approach is too simplified and does not reflect the true geometric characteristics of rockburst fragments.
Observation of large fragments (Figure 3(b)) shows that these fragments are mostly in the form of plates, sheets, or flakes. ReLiterature [17] proposed that the representative size of self-initiated rockburst fragments is length: width: thickness $=7.5: 5.0: 1.0$. The authors statistically analyzed the measurement data of a large number of seismically induced rockburst fragments and found that the length and width are close (length: width $=1-1.5$ ), and the thickness $(1-10 \mathrm{~mm})$ is much less than the length and width. Therefore, the large fragments are plate shaped with close length and width, and the thickness has little effect on the geometric feature of these fragments. Then, when calculating the equivalent size of fragments larger than $9.50 \mathrm{~mm}$, the thickness is ignored, and the equivalent size is chosen as the side length of a square with an area equal to the product of length and width. Based on the above analysis, the equivalent size of rockburst fragments can be calculated as

$$
R_{\mathrm{eq}}= \begin{cases}\frac{r_{1}+r_{2}}{2}, & R \leq 9.5 \mathrm{~mm}, \\ \sqrt{l w}, & R>9.5 \mathrm{~mm},\end{cases}
$$

where $R_{\mathrm{eq}}$ is the equivalent size and $R$ is the maximum size of a fragment in three directions; $r_{1}$ and $r_{2}$ are the diameters of the current and upper level sieves, respectively; and $l$ and $w$ are length and width, respectively.

\subsection{Classification, Characteristics, and Mass Distribution of} Rockburst Fragments. Different fragments are produced by macrofracture cutting of rock at different stages during the development of rockbursts. Therefore, the classification of fragments is helpful for understanding the fracturing mechanism of rockbursts. In addition, classification also helps to qualitatively estimate the fragmentation distribution and calculate the fractal dimensions of rockbursts. The fragments are classified into coarse, medium, fine, and micro 


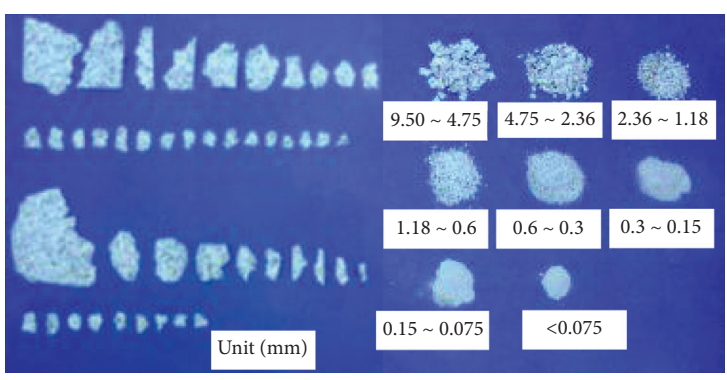

(a)

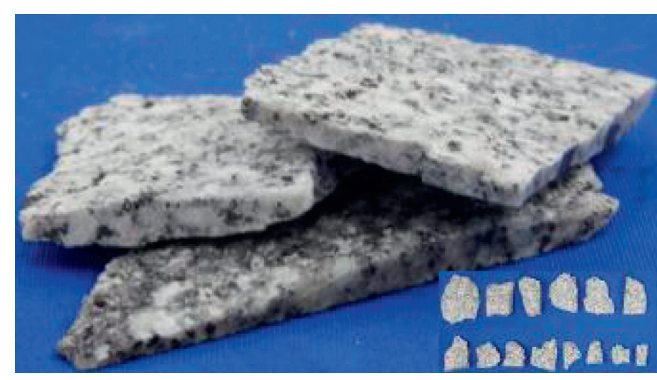

(b)

FIGURE 3: Characteristics of fragments generated from a seismically induced rockburst (sample CD-Z-3): (a) fragments distribution and (b) slab and slice shaped fragments.

grains, with the equivalent size falling in the ranges of $R_{\mathrm{eq}} \geq$ $40 \mathrm{~mm}, 4.75 \mathrm{~mm} \leq R_{\mathrm{eq}}<40 \mathrm{~mm}, 0.075 \mathrm{~mm} \leq R_{\mathrm{eq}}<4.75 \mathrm{~mm}$, and $R_{\text {eq }}<0.075 \mathrm{~mm}$, respectively.

According to Figure 3, the coarse grains are mostly plate, flake, or block shaped, which are mainly composed of rock slabs formed by splitting near the free face, blocks formed by breakage of rock slabs, and flakes formed by shearing in the burst pit $[4,9,34]$. The medium grains are mostly flake shaped with relatively small volume, which are mainly formed by shearing in the burst pit. The fine grains are mostly rice grain and fine sand shaped, which are mainly damaged from comminution of large fragments. The micro grains are powder shaped.

The cumulative mass distributions of different types of rockbursts are presented in Figure 4. It can be that the percentage of the micro grains is the smallest, that of the fine and medium grains is slightly larger, and that of the coarse grains is the largest. For different types of rockbursts, the mass percent of coarse grains is about $60 \%-70 \%$, and that of the moderate seismically induced rockbursts is the largest, that of the self-initiated rockbursts follows, and that of the weak seismically induced rockbursts is the lowest. For the moderate seismically induced rockbursts (Figures 4(b)$4(\mathrm{e}))$, the mass distribution is affected by the coupled static and disturbance conditions. That is, with the increase of static loads (Figures 4(b) and 4(c)) and disturbance amplitude (Figure $4(\mathrm{~d})$ ), the mass percent of fine and medium grains increases, whereas with the increase of disturbance frequency (Figure 4(e)), the mass percent of fine and medium grains decreases first and then increases. The increase of mass percent of fine and medium grains indicates that the distribution of rockburst fragments is more uniform and the degree of fragmentation is higher.

4.3. Fractal Dimension Calculation of Fragmentation Distribution. The fractal dimension of fragmentation distribution can be calculated according to the equivalent sizefrequency and mass-frequency relationships [13, 31]:

$$
\begin{gathered}
N=N_{0}\left(\frac{R_{\mathrm{eq}}}{R_{\max }}\right)^{-D}, \quad N=C_{1} R_{\mathrm{eq}}^{-D}, \\
N^{\prime}=N_{0}^{\prime}\left(\frac{M}{M_{\max }}\right)^{-b}, \quad N^{\prime}=C_{2} M^{-b},
\end{gathered}
$$

where, in equation (2), $N$ is the number of fragments with an equivalent size equal to or larger than $R_{\text {eq }}, N_{0}$ is the number of fragments with the maximum equivalent size of $R_{\max }, D$ is the fractal dimension, and $C_{1}$ is a constant; in equation (3), $N^{\prime}$ is the number of fragments with a mass equal to or larger than $M, N_{0}^{\prime}$ is the number of fragments with the maximum mass of $M_{\max }, b$ is the mass-frequency distribution index, and $C_{2}$ is a constant. Since the mass and equivalent size of a fragment satisfy $M \propto R_{\text {eq }}^{3}$, according to equations (2) and (3), we have

$$
D=3 b \text {. }
$$

Due to the fact that there are a large number of fragments and many of them are powder shaped, it is difficult to accurately determine the number of rockburst fragments. It is usually difficult to directly calculate the fractal dimension according to equation (2) or (3). The mass of fragments is easy to measure. It will be an effective way to calculate the fractal dimension if we can derive the mass-equivalent size relationship from equations (2) and (3). The derivation process is described as follows.

Let $M\left(R_{\mathrm{eq}}\right)$ represent the cumulative mass of fragments with equivalent size less than or equal to $R_{\text {eq }}, M$ represents the mass of fragments with equivalent size larger than $R_{\text {eq }}$, and $M_{T}$ represents the total mass of all fragments. In general, the percent of the cumulative mass of fragments approximately follows the Weibull distribution:

$$
\frac{M\left(R_{\mathrm{eq}}\right)}{M_{T}}=1-\exp \left[-\left(\frac{R_{\mathrm{eq}}}{\beta}\right)^{\alpha}\right]
$$




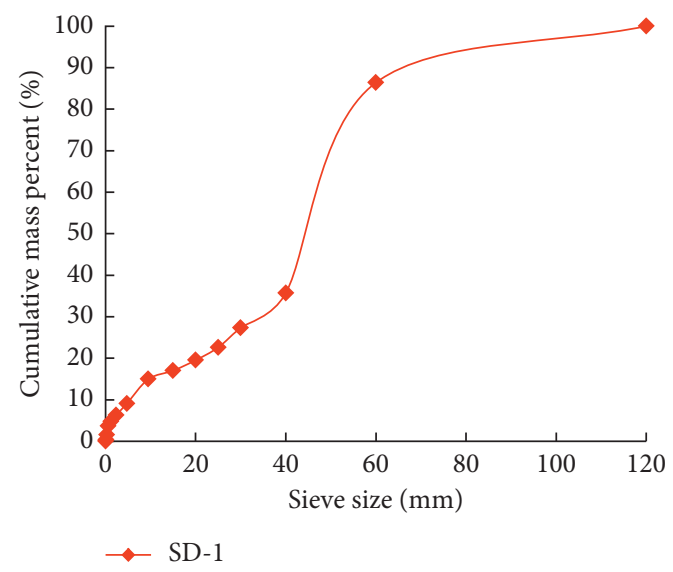

(a)

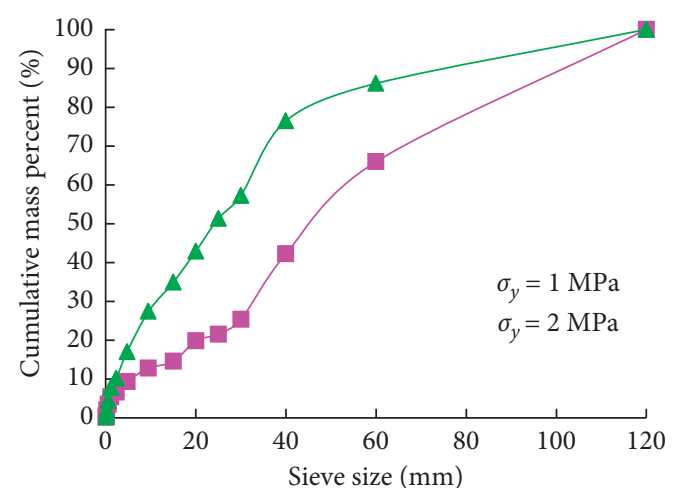

- $\mathrm{CD}-\mathrm{Y}-1$

$\leftarrow$ CD-Y-2

(c)

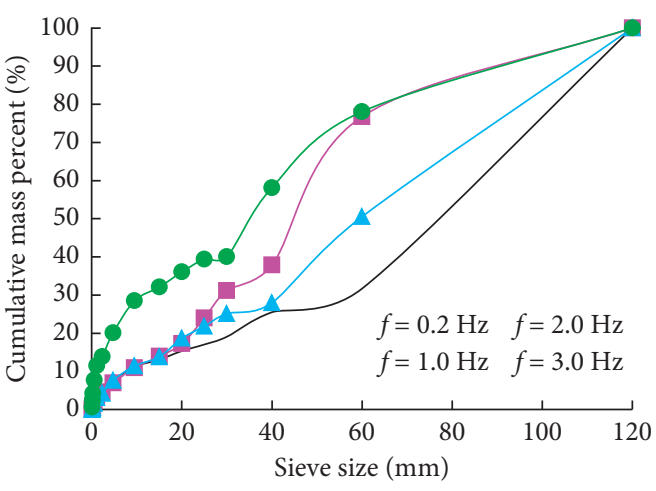

$-\mathrm{CD}-\mathrm{F}-1$
$-\mathrm{CD}-\mathrm{F}-2$

$$
\rightarrow \text { CD-F-3 }
$$$$
\text { - } \mathrm{CD}-\mathrm{F}-4
$$

(e)

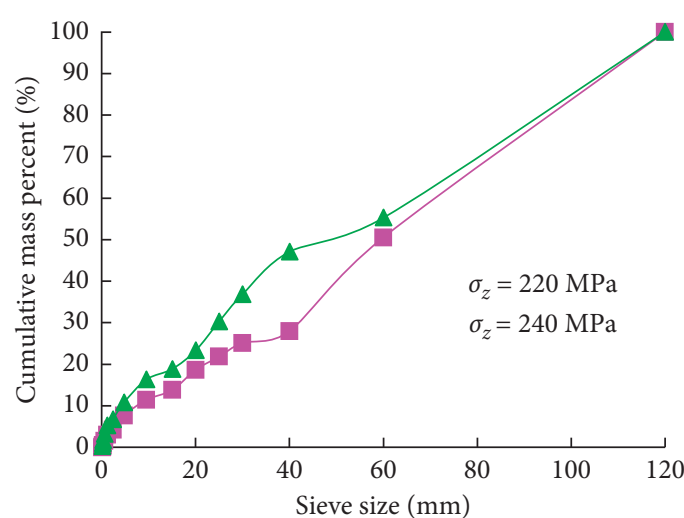

CD-Z-3

CD-Z-4

(b)

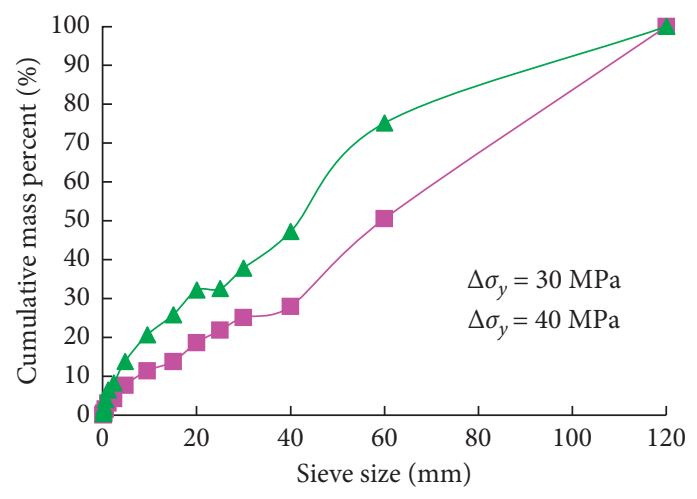

$\rightarrow$ CD-A-3

$\rightarrow$ CD-A-4

(d)

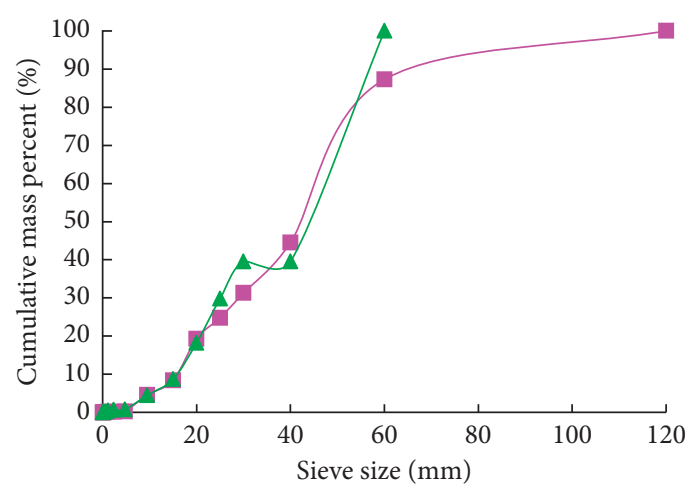

WD-1

WD-2

FIGURE 4: Cumulative mass distribution of fragments of different types of rockbursts: (a) the self-initiated rockbursts; (b-e) the moderate seismically induced rockbursts, (b) the maximum static stress $\sigma_{z}$ test group, (c) the minimum static stress $\sigma_{y}$ test group, (d) the disturbance amplitude $\Delta \sigma$ test group, and (e) the disturbance frequency $f$ test group; and (f) the weak seismically induced rockbursts.

where $\alpha$ is the shape parameters and $\beta$ is a parameter related to the average equivalent size. If $\left(R_{\mathrm{eq}} / \beta\right) \ll 1$, then equation (5) can be rewritten as

$$
\frac{M\left(R_{\mathrm{eq}}\right)}{M_{T}}=\left(\frac{R_{\mathrm{eq}}}{\beta}\right)^{\alpha} .
$$


Taking derivatives with respect to $R_{\mathrm{eq}}$ on both sides of equation (6), we have

$$
\mathrm{d} M\left(R_{\mathrm{eq}}\right) \propto\left(R_{\mathrm{eq}}\right)^{\alpha-1} \mathrm{~d} R_{\mathrm{eq}}
$$

Because $M=M_{T}-M\left(R_{\mathrm{eq}}\right)$ and $M_{T}$ is a constant,

$$
\mathrm{d} M=-\mathrm{d} M\left(R_{\mathrm{eq}}\right) \propto\left(R_{\mathrm{eq}}\right)^{\alpha-1} \mathrm{~d} R_{\mathrm{eq}} .
$$

Since $M$ is the mass of fragments with equivalent size larger than $R_{\text {eq }}$, then

$$
M=\sum_{R>R_{\mathrm{eq}}} m_{i}\left(R_{\mathrm{eq}, i}\right) N_{i} \propto \sum_{R>R_{\mathrm{eq}}} R_{\mathrm{eq}, i}^{3} N_{i},
$$

where $m_{i}\left(R_{\mathrm{eq}, i}\right)$ and $N_{i}$ are the mass and number of $i$ th group of fragments with equivalent size larger than $R_{\mathrm{eq}}$. Therefore, a general formula can be concluded from equation (9):

$$
\mathrm{d} M \propto R_{\mathrm{eq}}^{3} \mathrm{~d} N
$$

On the other hand, according to equation (2), we have

$$
\mathrm{d} N \propto R_{\mathrm{eq}}^{-D-1} \mathrm{~d} R_{\mathrm{eq}} .
$$

Substituting equation (11) into equation (10), we have

$$
\mathrm{d} M \propto R_{\mathrm{eq}}^{3-D-1} \mathrm{~d} R_{\mathrm{eq}}
$$

Comparison of equations (8) and (12) gives

$$
D=3-\alpha,
$$

Then, the fractal dimension of fragmentation distribution of rockbursts can be calculated according to the massequivalent size relationship (equation (13)). To illustrate the meaning of parameter $\alpha$, take natural logarithms of both sides of equation (6); we have

$$
\ln \left[\frac{M\left(R_{\mathrm{eq}}\right)}{M_{T}}\right]=\alpha \ln R_{\mathrm{eq}}-\alpha \ln \beta .
$$

It can be seen from equation (14) that if $\ln R_{\mathrm{eq}}$ is taken as the abscissa and $\ln \left[M\left(R_{\mathrm{eq}}\right) / M_{T}\right]$ is taken as the ordinate, then the $\ln \left[M\left(R_{\mathrm{eq}}\right) / M_{T}\right] \sim \ln R_{\mathrm{eq}}$ curve is a straight line, and the slope of this straight line is the parameter $\alpha$.

Once the fractal dimension is calculated, it can be used to quantitatively evaluate the characteristics of rockbursts [15-24]. In general, the larger the fractal dimension, the larger the number and the smaller the size of rockburst fragments, the greater the fragmentation degree of rockbursts, and the stronger the brittleness (i.e., the rockburst intensity) of rockbursts.

\section{Results and Discussion}

In this section, fractal dimensions of fragmentation distribution of different types of rockbursts are presented. The effects of different static and disturbance factors on the fractal dimensions of the moderate seismically induced rockbursts are investigated. Then, the fractal dimensions of different types of rockbursts are compared, and a quantitative relationship between the fractal dimension and kinetic energy of these types of rockbursts is established. Finally, based on the fractal analysis, the characteristics and mechanisms of different types of rockbursts are discussed.

\subsection{Fractal Dimensions of Fragmentation Distribution of the Moderate Seismically Induced Rockbursts}

5.1.1. Fractal Dimensions under Different Maximum Static Stress $\sigma_{z}$. Figure 5 shows the calculation results of fractal dimensions of the moderate seismically induced rockbursts under different maximum static stress $\sigma_{z}$. It can be seen that the fractal dimension increases with the increase of $\sigma_{z}$. This means that, under the preset high static stress, increasing the maximum static stress $\sigma_{z}$ can effectively improve the utilization of the energy input by the cyclic disturbance, so that the rock consumes more energy during the failure process, thus leading to a higher fragmentation degree.

5.1.2. Fractal Dimensions under Different Minimum Static Stress $\sigma_{y}$. Figure 6 shows the calculation results of fractal dimensions of the moderate seismically induced rockbursts under different minimum static stress $\sigma_{y}$. It can be seen that, as $\sigma_{y}$ increases, the fractal dimension increases. This shows that, under the conditions of low minimum stress $\sigma_{y}$ (1-5 $\mathrm{MPa})$ and rockburst occurrence, increasing $\sigma_{y}$ can strengthen the restraint of the rock and increase its strength, so that the rock can make full use of the energy input by the cyclic disturbance, thus leading to a higher fragmentation degree.

5.1.3. Fractal Dimensions under Different Disturbance Amplitude $\Delta \sigma$. Figure 7 shows the calculation results of fractal dimensions of the moderate seismically induced rockbursts under different disturbance amplitude $\Delta \sigma$. It can be seen that the fractal dimension increases as the disturbance amplitude $\Delta \sigma$ increases. Therefore, an increase of disturbance amplitude $\Delta \sigma$ can aggravate the fragmentation degree of rockbursts.

5.1.4. Fractal Dimensions under Different Disturbance Frequency $f$. Figure 8 shows the calculation results of fractal dimensions of the moderate seismically induced rockbursts under different disturbance frequency $f$. It can be seen that, in the range of $0.2-3 \mathrm{~Hz}$, as the disturbance frequency increases, the fractal dimension decreases first and then increases; that is, the fragmentation degree decreases first and then increases.

5.2. Fractal Dimensions of Fragmentation Distribution of the Weak Seismically Induced Rockbursts. Figure 9 shows the calculation results of fractal dimensions of the weak seismically induced rockbursts. It can be seen that the fractal 


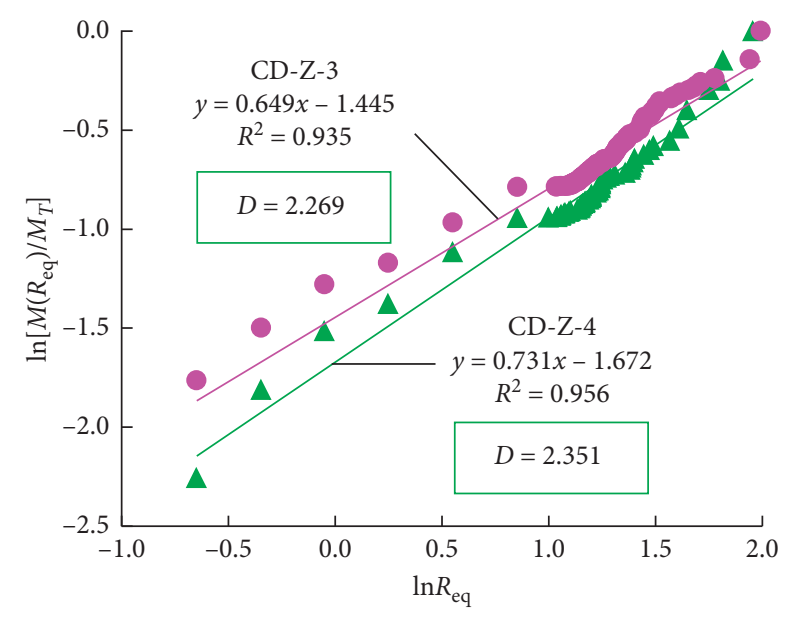

Figure 5: Calculation of fractal dimensions under different maximum static stress $\sigma_{z}$.

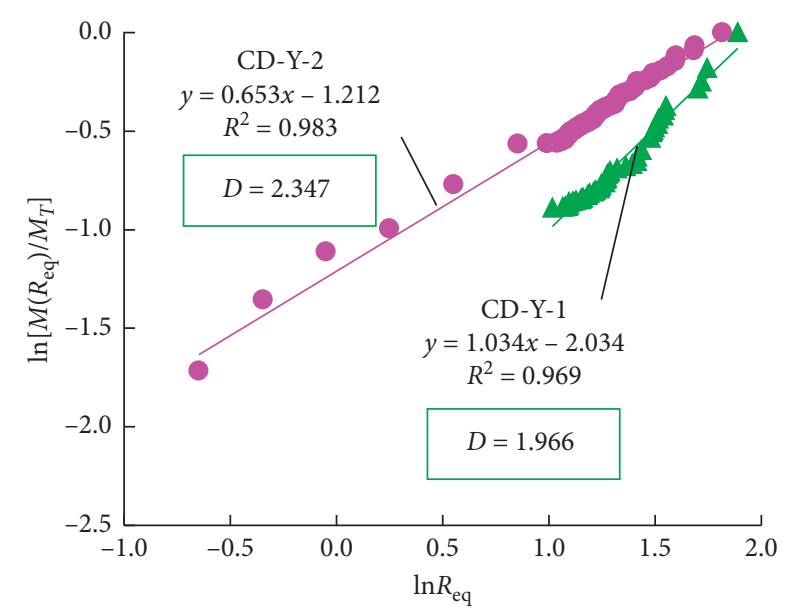

FIGURE 6: Calculation of fractal dimensions under different minimum static stress $\sigma_{y}$.

dimension increases with increasing minimum static stress, which is similar to that of the moderate seismically induced rockbursts (Figure 6). It is noted that the maximum fractal dimension of the weak seismically induced rockbursts is 1.802 , which is much lower than that of the moderate seismically induced rockbursts (mainly in the range of 2.3-2.5). The reason is that these two seismically induced rockbursts are controlled by different failure mechanisms, which will be explained in detail in Section 5.3.

\subsection{Discussion on Rockburst Mechanisms from Aspect of Fractal Analysis}

5.3.1. Comparison of Fractal Dimensions of Different Types of Rockbursts. The fractal dimension comprehensively reflects the quantity, size, uniformity of the distribution of rockburst fragments, and the brittleness of rockbursts. Therefore, a

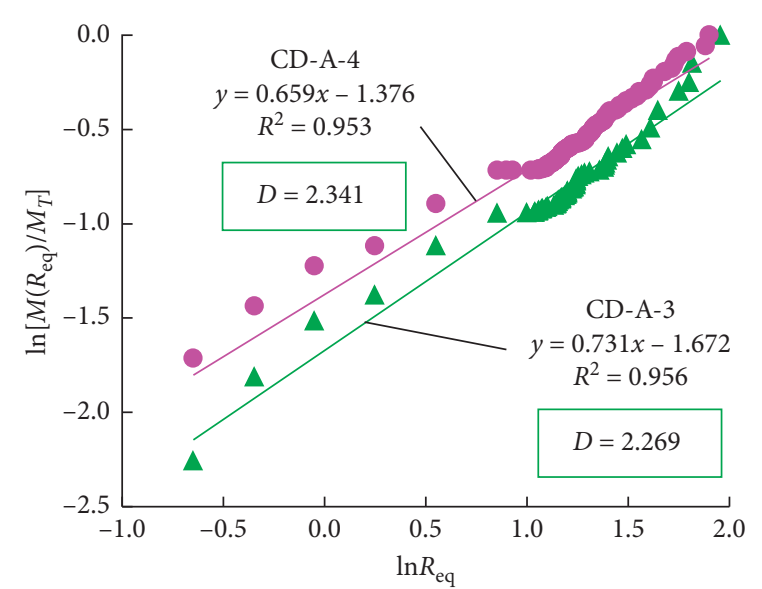

Figure 7: Calculation of fractal dimensions under different disturbance amplitude $\Delta \sigma$.

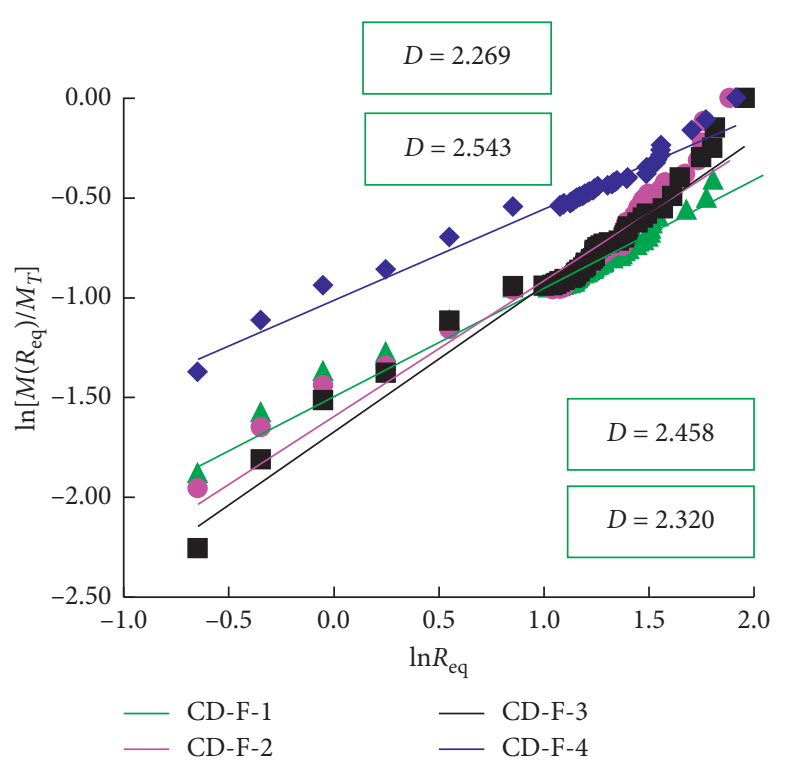

FIGURE 8: Calculation of fractal dimensions under different disturbance frequency $f$.

comparison of the fractal dimension is helpful for understanding the characteristics and mechanisms of different types of rockbursts. The calculation result of a typical selfinitiated rockburst is shown in Figure $10(D=1.733)$. The calculation results of fractal dimensions of different types of rockbursts studied in this study and those in the literature are presented in Table 2 and Figure 11. It can be seen that the fractal dimensions of different types of rockbursts fall in different ranges. According to the statistical results, the fractal dimension of the self-initiated rockbursts falls in the range of 1.7-2.2, that of the moderate seismically induced rockbursts falls in the range of 2.2-2.6, and that of the weak seismically induced rockbursts falls in the range of 1.0-1.8. Therefore, the fractal dimension of the moderate seismically induced rockbursts is larger than that of the self-initiated 


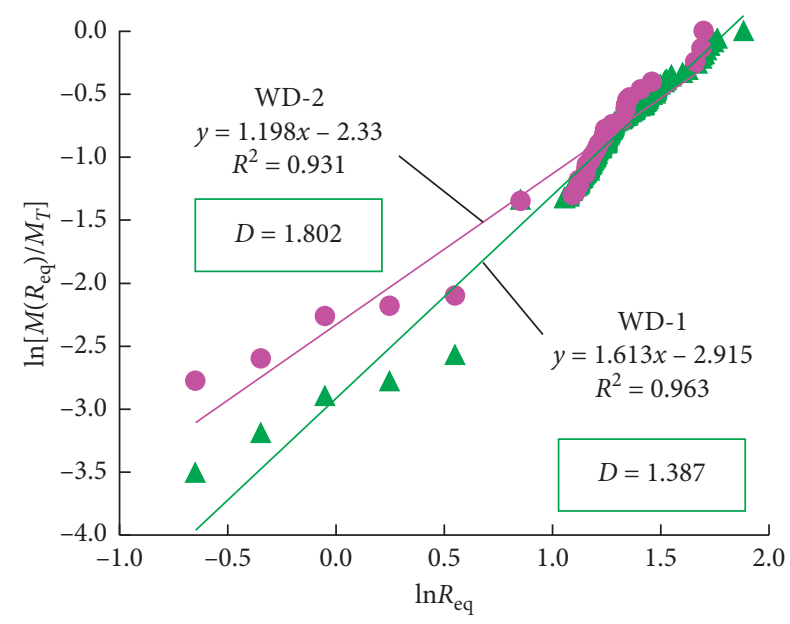

FIGURE 9: Calculation of fractal dimensions of the weak seismically induced rockbursts.

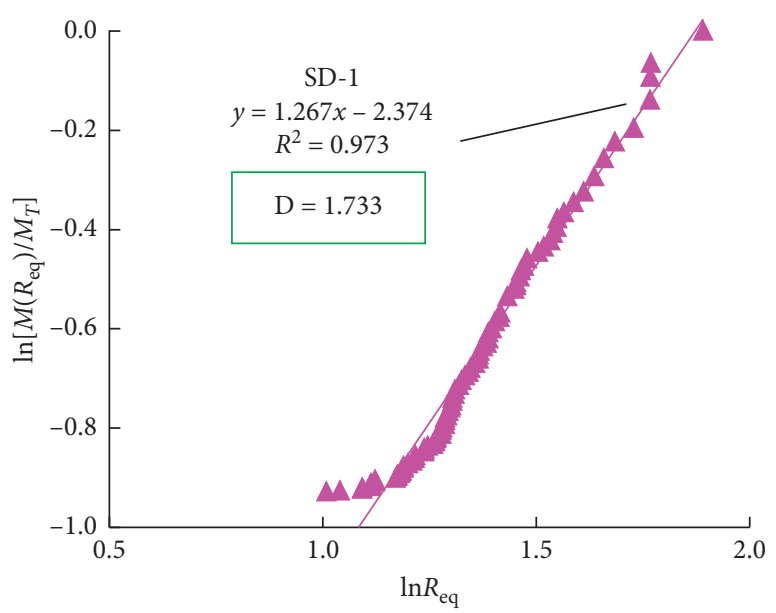

Figure 10: Calculation of fractal dimension of a typical self-initiated rockburst.

TABLE 2: Fractal dimensions of different types of rockbursts.

\begin{tabular}{lcc}
\hline Type of rockburst & Data source & Fractal dimension \\
\hline & $*$ & 1.733 \\
Self-initiated & Chen et al., 2019 & $1.830,1.880,1.970,2.020$, \\
rockbursts & [15] & $2.060,2.130,2.220$ \\
& Li et al., 2010 & $1.440,1.656,1.661,1.680$, \\
& [17], and Li & $1.710,1.783$ \\
\hline \multirow{3}{*}{$\begin{array}{l}\text { Moderate } \\
\text { seismically induced } 2014[18]\end{array}$} & Wang et al., & $1.770,1.920,2.050,2.240$, \\
rockbursts & 2019 [23] & $2.350,2.720,2.730,2.780$, \\
& $*$ & $2.900,2.930,2.950$ \\
\hline Weak seismically & Liu et al., 2014 & $1.387,1.802$ \\
induced rockbursts & {$[19]$} & $1.071,1.215,1.968$ \\
\hline
\end{tabular}

* denotes that the data are presented in this paper.

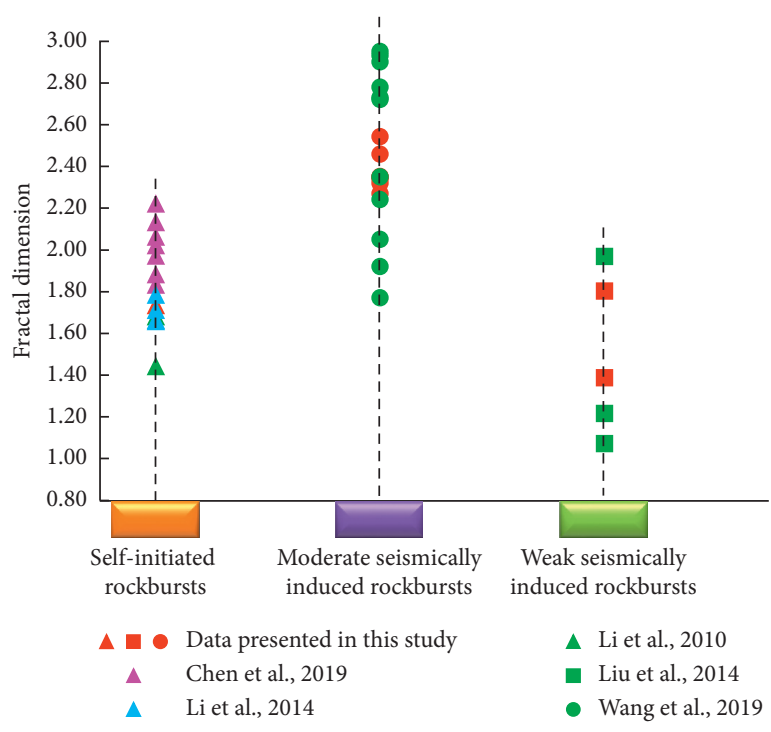

Figure 11: Comparison of the fractal dimensions of different types of rockbursts.

rockbursts, and the fractal dimension of the self-initiated rockbursts is larger than that of the weak seismically induced rockbursts.

5.3.2. Characteristics and Mechanisms of Different Types of Rockbursts. The characteristics of different types of rockbursts can be deduced from the statistical results of their fractal dimensions: the average fragment size of the moderate seismically induced rockbursts is the smallest, followed by that of the self-initiated rockbursts, and that of the weak seismically induced rockbursts is the largest; the number of fragments of the moderate seismically induced rockbursts is the largest, followed by that of the self-initiated rockbursts, and that of the weak seismically induced rockbursts is the least; the distribution of fragments of the moderate seismically induced rockbursts is the most uniform, followed by that of the self-initiated rockbursts, and that of the weak seismically induced rockbursts is the least uniform; the brittleness (i.e., the intensity) of the moderate seismically induced rockbursts is the strongest, followed by that of the self-initiated rockbursts, and that of the weak seismically induced rockbursts is the weakest.

The characteristics of different types of rockbursts can also be reflected in their failure modes (Figure 12). According to Figures 12(a) and 12(b) and previous studies $[4,9,27,28]$, the failure modes of the moderate seismically induced and the self-initiated rockbursts can be described as follows: most close to the free face, rock slabs are formed by tensile dominated splitting, a V-shaped or step-shaped rockburst pit is formed near the free face by combined tensile and shear failures, and several oblique macro shear bands are formed away from the free face. However, according to Figure 12(c) and literature $[4,5]$, the weak seismically induced rockbursts mainly undergo tensile splitting failure, and the rock sample is cut into several large thick rock slabs along the vertical direction. 


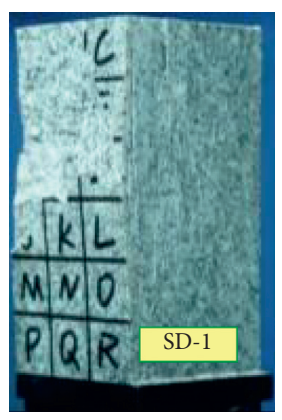

(a)

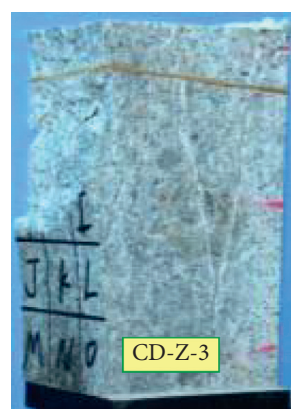

(b)
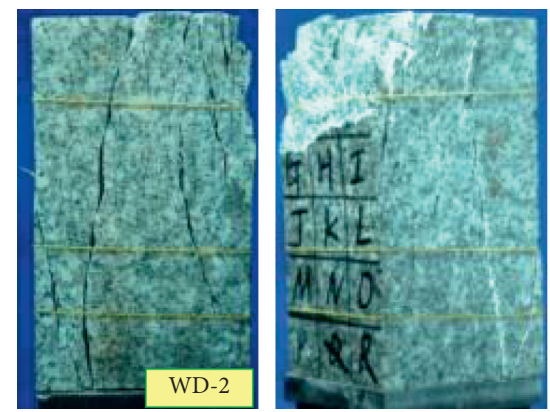

(c)

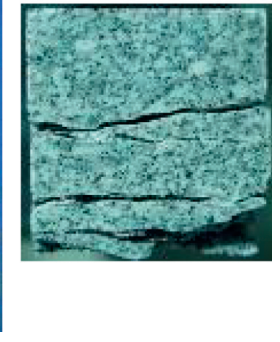

Figure 12: Comparison of typical failure patterns of rock samples of different types of rockbursts: (a) the self-initiated rockburst, (b) the moderate seismically induced rockburst, and (c) the weak seismically induced rockburst.

Microscopically, tensile cracks usually correspond to intergranular failure and shear cracks usually correspond to transgranular failure $[16,18,20,32]$. Macroscopically, tensile cracks are usually vertical and straight, and shear cracks are usually oblique and irregular [5, 28, 32, 35]. Therefore, from the perspective of fractal geometry, the fractal dimension of the relatively simpler tensile cracks is less than that of shear cracks. Then, based on the above analysis, it can be deduced that the weak seismically induced rockbursts are mainly controlled by tensile failure mechanism, the self-initiated and the moderate seismically induced rockbursts are controlled by combined tensile and shear failure mechanisms, and the shear failure characteristic of the moderate seismically induced rockbursts is more remarkable.

As a special form of dynamic failure, the dangerousness of rockbursts mainly comes from the kinetic energy of the ejected fragments. Kinetic energy is also an important reference index for selecting support measures $[12,36]$. In addition, the purpose of studying the fractal characteristics of rockbursts is not only to study the rockburst mechanisms but also to apply them in engineering practice. Therefore, it is necessary to link the fractal dimension with important physical quantities (e.g., the kinetic energy) of rockbursts. Figure 13 displays the data of kinetic energy and fractal dimension of different types of rockbursts. It can be seen that, due to the variation of test conditions and test errors, there is a certain degree of dispersion of the data. However, from a statistical point of view, under similar static loading conditions, the kinetic energy of the moderate seismically induced rockbursts is larger than that of the self-initiated rockbursts, and the kinetic energy of the self-initiated rockbursts is larger than that of the weak seismically induced rockbursts (Figure 13(a)). Furthermore, Figure 13(c) shows that there is a good linear relationship between the average fractal dimension and the kinetic energy of different types of rockbursts.

The linear relationship shown in Figure 13(c) is of great significance for studying the mechanisms of rockbursts. A rockburst is a complex nonlinear process if it is investigated from the perspectives of stress and deformation $[5,9,27,28]$. However, the problem can be simplified from an energy perspective. For example, Gong et al. [37] observed the linear energy storage and dissipation laws for different rocks under uniaxial compression. For rockbursts, the entire energy process is more complicated, which includes the energy input, storage, and dissipation in the prepeak stage and the energy input, dissipation, and release (i.e., the kinetic energy) in the postpeak stage. Therefore, there are no such linear laws similar to those proposed by Gong et al. [37] for the entire energy process of rockbursts $[4,38]$. However, from a fractal point of view, the problem will turn around. For example, during the experimental study of energy dissipation of rockbursts, Chen et al. [15] found that there is a good linear relationship between the fractal dimension and the total dissipated energy. Therefore, from the fractal point of view, the energy behaviors of rock during the entire rockburst process can be linearly described as the following:

$$
\begin{aligned}
& E_{\text {in }}=E_{\text {er }}+E_{p}+E_{k}, \\
& E_{p} \propto k_{1} D, \\
& E_{k} \propto k_{2} D,
\end{aligned}
$$

where $E_{\text {in }}$ is the total energy input to the rock, $E_{\text {er }}$ is the residual elastic energy, $E_{p}$ is the total dissipated energy, $E_{k}$ is the kinetic energy, $D$ is the fractal dimension, and $k_{1}$ and $k_{2}$ are constants. Since $E_{\text {er }}$ is a very small quantity that can be treated as a constant $[4,5,9]$, we have

$$
E_{\text {in }} \approx\left(k_{1}+k_{2}\right) D .
$$

Equations (15) and (16) indicate that, from the perspective of fractal, the energy input, dissipation, and release of rockbursts are all linear processes. The laws revealed by equations (15) and (16) can also provide a potential method for quantitative estimation of rockburst intensity in engineering practice. Generally speaking, in engineering practice, it is difficult to directly and quantitatively measure the kinetic energy of a rockburst, but semiquantitatively estimate the rockburst intensity based on the mass and ejection distance of the ejected fragments. Fortunately, the fractal dimension of fragmentation distribution of rockbursts studied in this study is a quantitative description of the mass 


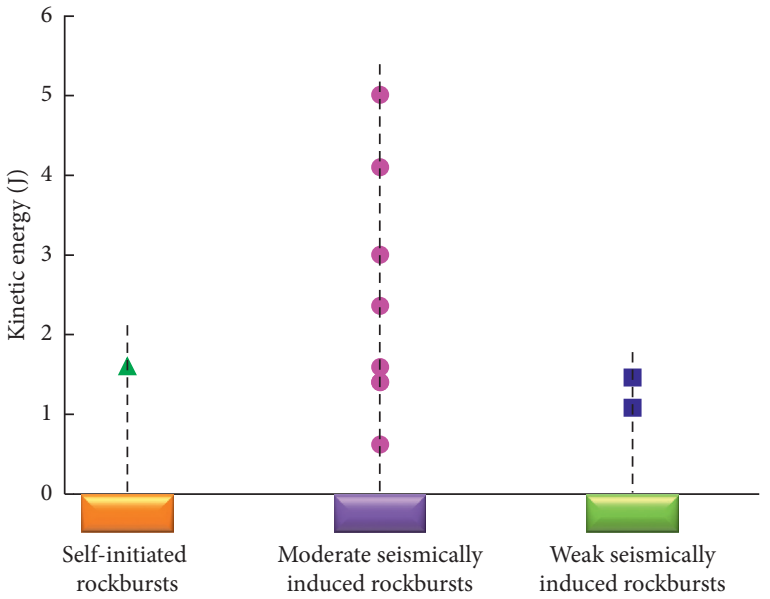

(a)

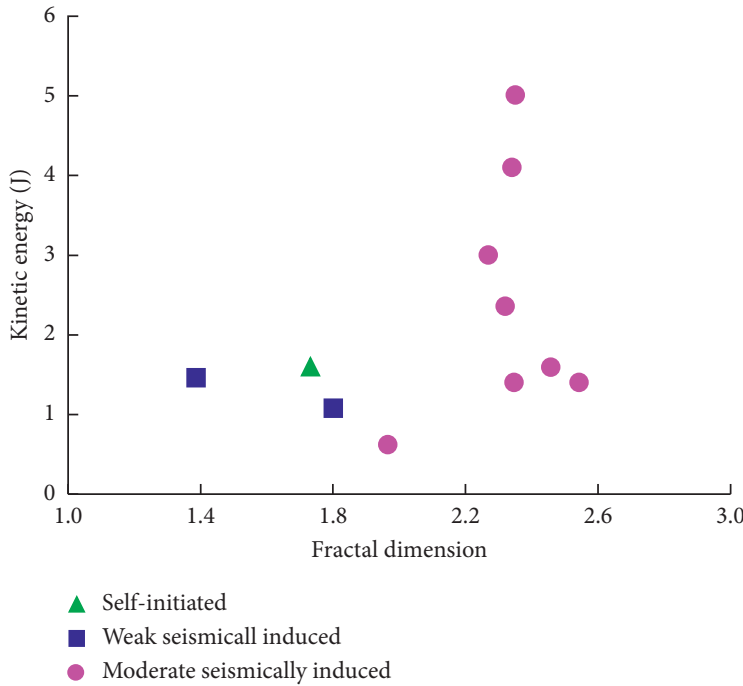

(b)

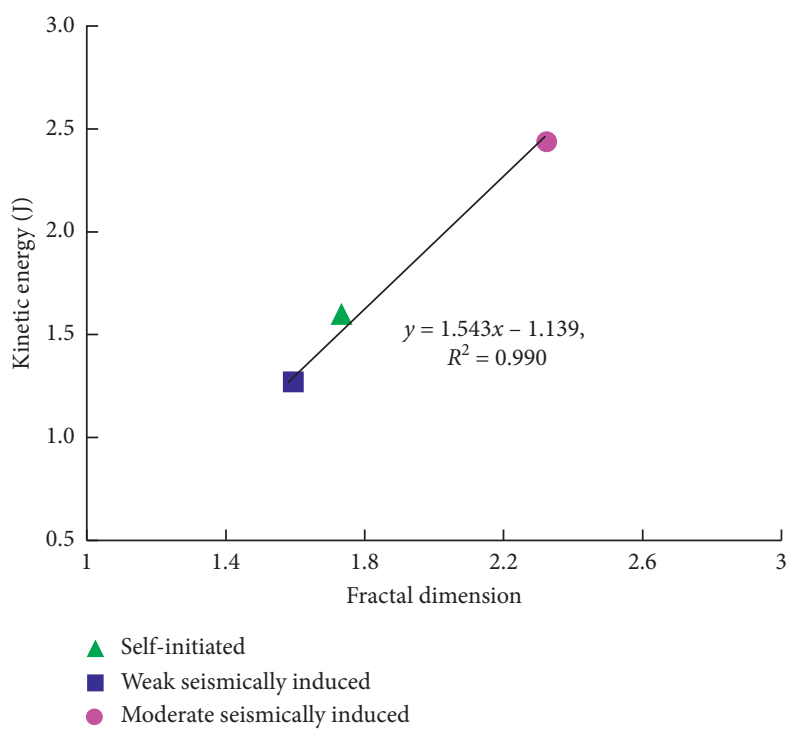

(c)

FigURE 13: Kinetic energy and relationship between fractal dimension and kinetic energy of different types of rockbursts: (a) kinetic energy, (b) scatter plots of fractal dimension and kinetic energy, and (c) linear relationship between the averaged fractal dimension and kinetic energy.

and ejection distance (related to the fracturing process) of rockburst fragments. Therefore, according to the linear relationship between fractal dimension and kinetic of rockbursts (equation (15)), the fractal dimension can be used as an effective indicator for quantitatively estimating the intensity of in situ rockbursts.

It should be noted that the observed linear law between the fractal dimension and kinetic energy is suitable for the three types of rockbursts studied in this paper. These three types of rockbursts are essentially three different forms of rock failures $[4,5,9]$, and their fractal dimensions and kinetic energies are distributed in distinct ranges. However, for a definite type of rockbursts, the fractal dimensions and kinetic energies are distributed in a rather narrow range (Figure 13), due to the variation of test conditions and test errors, there is yet no sufficient evidence supporting a linear relationship between the fractal dimension and kinetic energy. Further studies are needed to figure out the quantitative relationship between the fractal dimension and kinetic energy of rockbursts.

\section{Conclusions}

In the present study, the fractal characteristics of fragmentation distribution and mechanisms of the seismically induced rockbursts were investigated. The following conclusions can be drawn:

(1) The fragmentation distributions of both the seismically induced and self-initiated rockbursts exhibit fractal behaviors. The fractal dimension of the 
moderate seismically induced rockbursts is affected by the coupled static and disturbance conditions, that is, as the static stresses (i.e., the maximum and minimum static stresses) and disturbance amplitude increase, the fractal dimension increases, whereas as the disturbance frequency increases, the fractal dimension decreases first and then increases.

(2) From a statistical point of view, under similar static loading conditions, the moderate seismically induced rockbursts have the largest fractal dimension, followed by the self-initiated rockbursts, and the weak seismically induced rockbursts have the smallest fractal dimension.

(3) It can be deduced from the comparison of the fractal dimensions of the three types of rockbursts that the weak seismically induced rockburst is tensile splitting failure dominated, the self-initiated and the moderate seismically induced rockbursts are combined tensile-shear failures dominated, and the shear failure characteristic of the moderate seismically induced rockburst is more remarkable.

(4) There is a linear relationship between the average fractal dimension and kinetic energy of the three types of rockbursts, which implies that the fractal dimension can serve as an indicator for quantitatively estimating rockburst intensity. Furthermore, from a fractal point of view, the energy input, dissipation, and release (kinetic energy) of these three types of rockbursts are all linear processes.

\section{Data Availability}

The data used to support the findings of this study are available from the corresponding author upon request.

\section{Conflicts of Interest}

The authors declare that there are no conflicts of interest regarding the publication of this paper.

\section{Acknowledgments}

This research was funded by the National Natural Science Foundation of China (nos. 52009016 and 51909026) and the National Key Research and Development Plan of China (no. 2018YFC1505301).

\section{References}

[1] X. T. Feng, B. R. Chen, G. L. Feng, Z. N. Zhou, and H. Zheng, "Description of rockbursts in tunnels," Rockburst: Mechanisms, Monitoring, Warning and Mitigation, ButterworthHeinemann, Oxford, UK, 2018.

[2] K. Du, M. Tao, X.-B. Li, and J. Zhou, "Experimental study of slabbing and rockburst induced by true-triaxial unloading and local dynamic disturbance," Rock Mechanics and Rock Engineering, vol. 49, no. 9, pp. 3437-3453, 2016.

[3] M. He, H. Xia, X. Jia, W. Gong, F. Zhao, and K. Liang, "Studies on classification, criteria and control of rockbursts," Journal of
Rock Mechanics and Geotechnical Engineering, vol. 4, no. 2, pp. 97-114, 2012.

[4] L. H. Hu, "Study on the process of remotely triggered rockbursts induced by dynamic disturbance with low strain rates," Ph. D. Thesis, Guangxi University Nanning, China, 2017, in Chinese.

[5] L. Hu, K. Ma, X. Liang, C. Tang, Z. Wang, and L. Yan, "Experimental and numerical study on rockburst triggered by tangential weak cyclic dynamic disturbance under true triaxial conditions," Tunnelling and Underground Space Technology, vol. 81, pp. 602-618, 2018.

[6] J. Q. Jiang, G. S. Su, X. H. Zhang, and X. T. Feng, "Effect of initial damage on remotely triggered rockburst in granite: an experimental study," Bulletin of Engineering Geology and the Environment, vol. 79, pp. 3175-3194, 2020.

[7] X. Li, F. Gong, M. Tao et al., "Failure mechanism and coupled static-dynamic loading theory in deep hard rock mining: a review," Journal of Rock Mechanics and Geotechnical Engineering, vol. 9, no. 4, pp. 767-782, 2017.

[8] G. Su, X. Feng, J. Wang, J. Jiang, and L. Hu, "Experimental study of remotely triggered rockburst induced by a tunnel axial dynamic disturbance under true-triaxial conditions," Rock Mechanics and Rock Engineering, vol. 50, no. 8, pp. 2207-2226, 2017.

[9] G. Su, L. Hu, X. Feng et al., “True triaxial experimental study of rockbursts induced by ramp and cyclic dynamic disturbances," Rock Mechanics and Rock Engineering, vol. 51, no. 4, pp. 1027-1045, 2017.

[10] G. Su, Z. Chen, J. W. Ju, B. Zhao, S. Yan, and Z. Yan, "Experimental study of the dynamically induced rockburst of a rock wall with double free faces," International Journal of Damage Mechanics, vol. 28, no. 4, pp. 611-637, 2019.

[11] C. Xu, X. Liu, E. Wang, Y. Zheng, and S. Wang, "Rockburst prediction and classification based on the ideal-point method of information theory," Tunnelling and Underground Space Technology, vol. 81, pp. 382-390, 2018.

[12] M. Cai and P. K. Kaiser, Rockburst Support Reference Book, Volume 1 Rockburst Phenomenon and Support Characteristics, MIRARCO-Mining Innovation, Laurentian University, Sudbury, Canada, 2018.

[13] H. Xie and H. W. Zhou, "Application of fractal theory to topcoal caving," Chaos, Solitons \& Fractals, vol. 36, no. 4, pp. 797-807, 2008.

[14] H. P. Xie, J. F. Liu, Y. Ju, J. Li, and L. Z. Xie, "Fractal property of spatial distribution of acoustic emissions during the failure process of bedded rock salt," International Journal of Rock Mechanics and Mining Sciences, vol. 48, no. 8, pp. 1344-1351, 2011.

[15] Z. Chen, G. Su, J. W. Ju, and J. Jiang, "Experimental study on energy dissipation of fragments during rockburst," Bulletin of Engineering Geology and the Environment, vol. 78, no. 7, pp. 5369-5386, 2019.

[16] M. He, W. Nie, L. Han, and L. Ling, "Microcrack analysis of Sanya grantite fragments from rockburst tests," Mining Science and Technology (China), vol. 20, no. 2, pp. 238-243, 2010.

[17] D. J. Li, X. N. Jia, J. L. Miao, and M. C. He, "Analysis of fractal characteristics of fragment from rockburst test of granite," Chinese Journal of Rock Mechanics and Engineering, vol. 29, no. 1, pp. 3280-3289, 2010, in Chinese.

[18] D. Li, F. Zhao, and M. Zheng, "Fractal characteristics of cracks and fragments generated in unloading rockburst tests," International Journal of Mining Science and Technology, vol. 24, no. 6, pp. 819-823, 2014. 
[19] D. Liu, D. Li, F. Zhao, and C. Wang, "Fragmentation characteristics analysis of sandstone fragments based on impact rockburst test," Journal of Rock Mechanics and Geotechnical Engineering, vol. 6, no. 3, pp. 251-256, 2014.

[20] M. C. He, F. Zhao, M. Cai, and S. Du, "A novel experimental technique to simulate pillar burst in laboratory," Rock Mechanics and Rock Engineering, vol. 48, no. 5, pp. 1-16, 2015.

[21] X.-T. Feng, Y. Yu, G.-L. Feng, Y.-X. Xiao, B.-R. Chen, and Q. Jiang, "Fractal behaviour of the microseismic energy associated with immediate rockbursts in deep, hard rock tunnels," Tunnelling and Underground Space Technology, vol. 51, pp. 98-107, 2016.

[22] G. Su, Y. Shi, X. Feng, J. Jiang, J. Zhang, and Q. Jiang, “Truetriaxial experimental study of the evolutionary features of the acoustic emissions and sounds of rockburst processes," Rock Mechanics and Rock Engineering, vol. 51, no. 2, pp. 375-389, 2017.

[23] Y. Wang, Z. Chun, M. He, D. Liu, Y. Lin, and S. Du, "Fragmentation characteristics analysis of sandstone fragments for impact rockburst under different dynamic loading frequency," Geotechnical and Geological Engineering, vol. 37, no. 4, pp. 2715-2727, 2019.

[24] Y. Yu, X. T. Feng, C. J. Xu, B. R. Chen, Y. X. Xiao, and G. L. Feng, "Spatial fractal structure of microseismic events for different types of rockburst in deeply buried tunnels," International Journal of Geomechanics, vol. 20, no. 4, p. $04020025,2020$.

[25] H. Sun, X. L. Liu, and J. B. Zhu, "Correlational fractal characterisation of stress and acoustic emission during coal and rock failure under multilevel dynamic loading," International Journal of Rock Mechanics and Mining Sciences, vol. 117, pp. 1-10, 2019.

[26] H. Sun, X. L. Liu, S. G. Zhang, and K. Nawnit, "Experimental investigation of acoustic emission and infrared radiation thermography of dynamic fracturing process of hard-rock pillar in extremely steep and thick coal seams," Engineering Fracture Mechanics, vol. 226, p. 106845, 2020.

[27] G. Su, J. Jiang, S. Zhai, and G. Zhang, "Influence of tunnel axis stress on strainburst: an experimental study," Rock Mechanics and Rock Engineering, vol. 50, no. 6, pp. 1551-1567, 2017.

[28] G. Su, S. Zhai, J. Jiang, G. Zhang, and L. Yan, "Influence of radial stress gradient on strainbursts: an experimental study," Rock Mechanics and Rock Engineering, vol. 50, no. 10, pp. 2659-2676, 2017.

[29] C. Zhang, X.-T. Feng, H. Zhou, S. Qiu, and W. Wu, "Case histories of four extremely intense rockbursts in deep tunnels," Rock Mechanics and Rock Engineering, vol. 45, no. 3, pp. 275-288, 2012.

[30] B. B. Mandelbrot, The Fractal Geometry of Nature, W. H. Freeman and Company, New York, NY, USA, 1982.

[31] H. P. Xie, Fractals in Rock Mechanics, CRC Press, Boca Raton, FL, USA, 1993.

[32] S. Zhai, G. Su, S. Yin, B. Zhao, and L. Yan, "Rockburst characteristics of several hard brittle rocks: a true triaxial experimental study," Journal of Rock Mechanics and Geotechnical Engineering, vol. 12, no. 2, pp. 279-296, 2020.

[33] S. Li, X.-T. Feng, Z. Li, B. Chen, C. Zhang, and H. Zhou, "In situ monitoring of rockburst nucleation and evolution in the deeply buried tunnels of Jinping II hydropower station," Engineering Geology, vol. 137-138, pp. 85-96, 2012.

[34] G. S. Su, L. H. Hu, X. T. Feng, J. H. Wang, and X. H. Zhang, "True triaxial experimental study of rockburst process under low frequency cyclic disturbance load combined with static load," Chinese Journal of Rock Mechanics and Engineering, vol. 35, no. 7, pp. 1309-1322, 2016, in Chinese.

[35] L. Hu, G. Su, X. Liang, Y. Li, and L. Yan, "A distinct element based two-stage-structural model for investigation of the development process and failure mechanism of strainburst," Computers and Geotechnics, vol. 118, p. 103333, 2020.

[36] P. K. Kaiser and M. Cai, "Design of rock support system under rockburst condition," Journal of Rock Mechanics and Geotechnical Engineering, vol. 4, no. 3, pp. 215-227, 2012.

[37] F. Gong, J. Yan, S. Luo, and X. Li, "Investigation on the linear energy storage and dissipation laws of rock materials under uniaxial compression," Rock Mechanics and Rock Engineering, vol. 52, no. 11, pp. 4237-4255, 2019.

[38] B. G. Tarasov and T. R. Stacey, "Features of the energy balance and fragmentation mechanisms at spontaneous failure of class I and class II rocks," Rock Mechanics and Rock Engineering, vol. 50, no. 10, pp. 2563-2584, 2017. 